Technical Report, 2004

\title{
Membrane Targeting of P-type ATPases in Plant Cells
}

\author{
PI: Jeffrey F. Harper, University of Nevada, Reno (transferred from The Scripps Research \\ Institute, June 2004)
}

\begin{abstract}
How membrane proteins are targeted to specific subcellular locations is a very complex and poorly understood area of research. Our long-term goal is to use P-type ATPases (ion pumps), in a model plant system Arabidopsis, as a paradigm to understand how members of a family of closely related membrane proteins can be targeted to different subcellular locations. The research is divided into two specific aims. The first aim is focussed on determining the targeting destination of all 10 ACA-type calcium pumps (Arabidopsis Calcium ATPase) in Arabidopsis. ACAs represent a plant specific-subfamily of plasma membrane-type calcium pumps. In contrast to animals, the plant homologs have been found in multiple membrane systems, including the ER (ACA2), tonoplast (ACA4) and plasma membrane (ACA8). Their high degree of similarity provides a unique opportunity to use a comparative approach to delineate the membrane specific targeting information for each pump. One hypothesis to be tested is that an endomembrane located ACA can be re-directed to the plasma membrane by including targeting information from a plasma membrane isoform, ACA8. Our approach is to engineer domain swaps between pumps and monitor the targeting of chimeric proteins in plant cells using a Green Fluorescence Protein (GFP) as a tag. The second aim is to test the hypothesis that heterologous transporters can be engineered into plants and targeted to the plasma membrane by fusing them to a plasma membrane proton pump. As a test case we are evaluating the targeting properties of fusions made between a yeast sodium/proton exchanger (Sod2) and a proton pump (AHA2). This fusion may potentially lead to a new strategy for engineering salt resistant plants. Together these aims are designed to provide fundamental insights into the biogenesis and function of plant cell membrane systems.
\end{abstract}

General. This report is to accompany the transfer of my DOE grant from the Scripps Research Institute to the University of Nevada Reno. While the lab transfer has interrupted the continuity of research on the above specific aims, considerable progress has been made, as outlined below:

\section{Publications.}

1. Schiøtt, M., S. Romanowsky, M. K. Jakobsen, L. Bækgaard, M.G. Palmgren, J.F. Harper. 2004. A plant plasma membrane $\mathrm{Ca}^{2+}$ pump is required for normal pollen tube growth and fertilization. PNAS 101: 9502-9507

\begin{abstract}
Ca}^{2+}$ signals are thought to play important roles in plant growth and development, including key aspects of pollen tube growth and fertilization. The dynamics of a $\mathrm{Ca}^{2+}$ signal are largely controlled by influx (through channels) and efflux (through pumps and antiporters). The Arabidopsis genome encodes $14 \mathrm{Ca}^{2+}$ pumps, ten of which belong to a family of autoinhibited $\mathrm{Ca}^{2+}$ ATPases (ACA) that are predicted to be activated by $\mathrm{Ca}^{2+} /$ calmodulin. Here we show that isoform ACA9 is expressed primarily in pollen and localized to the plasma membrane. Three independent T-DNA gene disruptions of ACA9 were found to result in partial male sterility. Complementation was observed using a ACA9YFP (yellow fluorescence protein) fusion that displayed plasma membrane localization. Mutant aca9 pollen displayed a reduced growth potential and a high frequency of aborted
\end{abstract}


fertilization resulting in a greater than $80 \%$ reduction in seed set. These findings identify a plasma membrane $\mathrm{Ca}^{2+}$ transporter as a key regulator of pollen development and fertilization in flowering plants.

2. The following manuscript has been submitted to PNAS. This report provides evidence that at least one of the plasma membrane P-type ATPases has a secondary endomembrane function.

A Plasma Membrane $\mathrm{H}^{+}$-ATPase Is Required for the Formation of Proanthocyanidins in the Seed Coat Endothelium of Arabidopsis thaliana

Ivan R. Baxter ${ }^{1 *}$, Jeffery C. Young ${ }^{2 *}$, Gordon Armstrong ${ }^{2}$, Nathan Foster ${ }^{2}$, Wendy Ann Peer ${ }^{1}$, Samuel P. Hazen ${ }^{4}$, Angus S. Murphy ${ }^{1}$, and Jeffrey F. Harper ${ }^{3,4}$

\begin{abstract}
The plasma membrane in plant cells is energized with an electrical potential and proton gradient generated through the action of $\mathrm{H}^{+}$-pumps belonging to the P-type ATPase superfamily. The Arabidopsis genome encodes 11 plasma membrane $\mathrm{H}^{+}$-pumps. Isoform AHA10 (autoinhibited $\mathrm{H}^{+}$-ATPase isoform 10) is expressed primarily in developing seeds. Here we show that four independent gene disruptions of AHA10 result in seed coats with a transparent testa phenotype (tt, light-colored seeds). A quantitative analysis of extractable flavonoids in aha10 seeds revealed an approximately 100-fold reduction of PA (proanthocyanidin), one of the two major end-product pigments in the flavonoid biosynthetic pathway. In wild-type seed coat endothelial cells, PA accumulates in a large central vacuole. In aha10 mutants, the formation of this vacuole is impaired, as indicated by the predominance of multiple small vacuoles observed by fluorescence microscopy using a vacuole-specific dye, DCFDA (5-(and -6)-carboxy 2',7'-dichlorofluorescein diacetate). Evidence that aha10 endothelial cells are otherwise healthy is indicated by no significant decrease in (i) the accumulation of other flavonoid pathway end products, such as anthocyanins, and (ii) mRNA levels for two endothelium-specific transcripts (TT12 and $B A N)$. Thus, the specific effect of aha10 on vacuolar and PA biogenesis provides unexpected evidence to support an endomembrane function for a member of the plasma membrane $\mathrm{H}^{+}$ATPase family.
\end{abstract}

3. The following manuscript has provided an improved foundation for our analysis of targeting information in P-type ATPases

Baxter I, Tchieu J, Sussman MR, Boutry M, Palmgren M, Gribskov M, Harper JF*, Axelsen KB (2003) Genomic Comparison of P-Type ATPase Ion Pumps in Arabidopsis and Rice. Plant Phys 132: 618-628. *JFH, Corresponding Author

4. The following manuscript provided the first in planta function and localization information for a heavy metal pump in plants.

Hussain,D., M J Haydon, Y Wang, E Wong, S.M. Sherson, J. Young, J. Camakaris, JF. Harper, C. S. Cobbett,. 2004. P-type ATPase heavy metal transporters with roles in essential zinc metabolism in Arabidopsis. Plant Cell 16: 1327-1339 